\title{
Delayed cortical gray matter development in neonates with severe congenital heart disease
}

\author{
Nathalie H.P. Claessens' ${ }^{1}$ Pim Moeskops ${ }^{1,2}$, Andreas Buchmann ${ }^{3}$, Beatrice Latal ${ }^{4,5}$, Walter Knirsch ${ }^{5,6}$, lanina Scheer ${ }^{5,7}$, \\ Ivana Išgum ${ }^{2}$, Linda S. de Vries', Manon J.N.L. Benders ${ }^{1}$ and Michael von Rhein ${ }^{4,5,8}$; on behalf of the Research \\ Group Heart and Brain
}

BACKGROUND: This study aimed to assess cortical gray matter growth and maturation in neonates with congenital heart disease (CHD).

METHODS: Thirty-one (near) term neonates with severe CHD (8 univentricular heart malformation (UVH), 21 d-transposition of great arteries ( $d$-TGA) and 2 aortic coarctation) underwent cerebral MRI before (postnatal-day 7) and after (postnatal-day 24) surgery. Eighteen controls with similar gestational age had one MRI (postnatal-day 23). Cortical gray matter volume (CGM), inner cortical surface (iCS), and median cortical thickness were extracted as measures of volumetric growth, and gyrification index (Gl) as measure of maturation.

RESULTS: Over a median of $18 \mathrm{~d}$, CGM increased by 21\%, iCS by $17 \%$, thickness and Gl both by $9 \%$. Decreased postoperative CGM and iCS were seen for CHD compared to controls ( $P$ values $<0.01)$, however with similar thickness and GI. UVH showed lower postoperative iCS, thickness ( $P$ values $<0.05$ ) and $\mathrm{Gl}(P$ value $<0.01)$ than $\mathrm{d}-\mathrm{TGA}$ and controls. Infants requiring preoperative balloon-atrioseptostomy (BAS, 61\%) had reduced postoperative CGM, iCS, and GI (P values < 0.05).

CONCLUSION: Infants with severe CHD show reduced cortical volumes compared to controls with gyrification being delayed in UVH, but not in d-TGA. Infants requiring BAS show higher risk of impaired cortical volume and gyrification.

$B^{\text {r }}$ rain lesions are a common finding in infants with severe congenital heart disease (CHD) affecting up to 50\% (1). This serious morbidity is associated with long-term neurodevelopmental sequelae, and as they grow older, many CHD patients show motor- and cognitive delays (2), as well as impairments in the domains of learning and behavior (3). Hypotension and hypoxemia before cardiopulmonary bypass surgery $(\mathrm{CPB})$ are substantial problems in $\mathrm{CHD}$, leading to reduced oxygen delivery to the brain $(1,4)$. This seems to increase the vulnerability of the brain to the subsequent openheart surgery requiring $\mathrm{CPB}(1)$.

The importance of brain imaging in CHD infants is becoming more widely accepted and magnetic resonance imaging (MRI) has proven to be a useful and accessible tool to detect and evaluate brain injury. Alterations in brain development in CHD patients have been described preoperatively (5), and even in utero $(6,7)$, with also new cerebral lesions found after the surgical procedure (8). But even in the absence of obvious abnormal MRI findings on qualitative assessment, quantitative measurements may find more subtle alterations. The negative effects of white matter abnormalities on neurodevelopment have been noticed in several studies $(9,10)$, but white matter developmental delays or alterations are known to disturb growth and neuronal maturation of the cortical gray matter as well $(11,12)$. The volumetric growth and complex gyrification of the cortex mainly takes place in the third trimester of pregnancy (13), and impacts cognition, motor, and behavioral functioning (14). Longitudinal, quantitative data on cortical gray matter pre- and postsurgery in neonates with CHD is still sparse, and additional knowledge might help in the prediction of long-term neurodevelopment.

The aim of this study was to assess the development of cortical gray matter in neonates undergoing cardiopulmonary bypass surgery for severe congenital heart disease, before and after surgery, in comparison to healthy term born controls at corresponding postoperative age. We hypothesized that cortical growth and maturation-presented as volume expansion and gyrification-between both assessments would be present, with a reduction of these parameters when compared to healthy neonates assessed at corresponding postoperative age. The second aim was to evaluate possible clinical risk factors influencing cortical development in this patient group, including birth characteristics, type and severity of $\mathrm{CHD}$, timing and procedure of surgery, and postoperative course.

\footnotetext{
The last two authors contributed equally to this work.

'Department of Neonatology, Wilhelmina Children's Hospital, University Medical Centre Utrecht, The Netherlands; ${ }^{2}$ Image Sciences Institute, University Medical Centre Utrecht, The Netherlands; ${ }^{3}$ Department of Neurology, University Hospital Zurich, Switzerland; ${ }^{4}$ Child Development Center, University Children's Hospital Zurich, Switzerland; ${ }^{5}$ Children's Research Center, University Children's Hospital, Zurich, Switzerland; ${ }^{6}$ Department of Cardiology, University Children's Hospital, Zurich, Switzerland; ${ }^{7}$ Diagnostic Imaging, University Children's Hospital, Zurich, Switzerland; ${ }^{8}$ Developmental Pediatrics, SPZ, Kantonsspital Winterthur, Switzerland. Correspondence: Manon J.N.L. Benders (m.benders@umcutrecht.nl)

Received 2 March 2016; accepted 15 May 2016; advance online publication 24 August 2016. doi:10.1038/pr.2016.145
} 


\section{RESULTS}

\section{Patients}

Table 1 shows the baseline characteristics of the study population. Thirty-one patients (68\% dextro-transposition of the great arteries (d-TGA), 26\% univentricular heart malformation (UVH), 7\% coarctation of the aorta (CoA) were included with a mean gestational age of $39.6 \mathrm{wk}$ and $74 \%$ male. Table 2 shows the perioperative details. Good quality brain segmentations were obtained preoperatively in 26 infants at a median postnatal age of $7 \mathrm{~d}$ (postmenstrual age (PMA) $40^{+5} \mathrm{wk}$ ) and postoperatively in 23 infants at median postnatal age of $24 \mathrm{~d}$ (PMA $43^{+1} \mathrm{wk}$ ). Eighteen healthy controls underwent MRI at a median postnatal age of $23 \mathrm{~d}$ (PMA $42^{+6} \mathrm{wk}$ ). For five infants preoperatively and eight infants postoperatively segmentations or MR images were of too poor quality for cortex assessment. No significant differences in baseline characteristics were seen between infants with and without available cortex assessment.

\section{Cortical Growth and Maturation}

For 18 infants, brain segmentations at both time points were available. All cortical parameters increased significantly from pre- to postoperative MRI ( $P$ values $<0.001$ for cortical gray matter (CGM) (+21\%), inner cortical surface $(i \mathrm{CS})(+17 \%)$, and gyrification index (GI) (+9\%), $P$ value $<0.01$ for thickness $(+9 \%))$, as shown in Figure 1 (first and fourth plot). Unmyelinated white matter (UWM) also increased between both time points $(P$ value $<0.05)$, but with a lower rate $(+5 \%)$. Infants with preoperative mild-moderate white matter injury $(17 \%)$ showed lower relative $(P$ value $=0.033, r=0.503)$ and absolute $(P$ value $=0.019, r=0.547)$ increase of gyrification index over this period. The d-TGA group (82\%) showed higher, but nonsignificant, absolute, and relative growth rates of all measured cortical parameters than the UVH group.

\section{Postoperative Cortical Morphology Compared to Healthy Controls}

For the infants with severe $\mathrm{CHD}$, reductions in postoperative CGM volumes $(-14 \%)$ compared to controls were found. Additionally, decreased $i$ CS (-12\%) was seen for CHD. However, median cortical thickness and GI were similar ( $P$ values 0.19 and 0.53 , respectively). This is also shown in Figure 1 (fourth and seventh plot). No significant left-right differences in cortical parameters could be found at any time point.

\section{Perinatal Risk Factors}

Table 3 shows the correlations between clinical risk factors and cortical parameters with correction for PMA. Infants with d-TGA showed significantly higher $i \mathrm{CS}$ at the postoperative scan than infants with UVH $(+8 \%)$, as well as larger cortical thickness $(+11 \%)$ and GI $(+5 \%)$. This can also be found in Figure 1 (preoperatively in second and third plot; postoperatively in fifth and sixth plot). As the CoA group was too small for analysis (two cases), only the d-TGA and UVH groups were compared with respect to cortical parameters. Lower birth weight was significantly associated with lower pre- and postoperative cortical volumetric parameters and borderline with lower gyrification index. Gestational age was only related to CGM at both time points. All significant relations persisted after correction for sex.

\section{Postnatal Risk Factors}

BAS was required in $62 \%$ of the d-TGA, $63 \%$ of the UVH, and $50 \%$ (one of two cases) of the CoA group. Smaller CGM volume $(-8 \%)$, iCS $(-5 \%)$, and GI $(-4 \%)$ on the postoperative scan were seen for infants undergoing BAS compared to infants who did not require this intervention, as also shown in Table 3. Infants with preoperative BAS showed significantly lower preoperative mean $\mathrm{O}_{2}$ saturation than those without ( 84 vs. $90 \%, P$ value $=0.002)$. Again, all correlations persisted after correction for sex. Infants with severe preoperative brain injury (white matter injury and/or ischemic stroke, 12\%) showed comparable cortical parameters as their peers without brain injury. No associations of peri- and postoperative risk factors with cortical parameters were found.

\section{DISCUSSION}

This study describes pre- and postoperative cortical gray matter volumetric growth and maturation in neonates with $\mathrm{CHD}$ undergoing cardiopulmonary bypass surgery. A significant increase of cortical volume and gyrification over time could be found, but postoperative cortical volume was reduced when compared to healthy term born controls. However, gyrification indices were not impaired-a sign of normal cortical maturation. In addition, a lower birth weight, a univentricular heart malformation, and preoperative requirement of balloon atrioseptostomy were risk factors of smaller cortical volume and impaired maturation after surgery. Lower gestational age was a risk factor for lower CGM volume, but not for cortical maturation.

This analysis extends the findings of previously published results on reduced postoperative brain volumes in this study cohort (15) and adds information on cortical growth and gyrification from pre- to postoperative MRI. Typical cortical development includes immense volumetric expansion in the third trimester of pregnancy, with gyrification starting around the 24th week of gestation in the central part of the brain and eventually developing into a brain folded similar to the adult brain around term $(13,16)$. In line with these findings, this study showed a remarkable increase of cortical volume and maturation in the median $18 \mathrm{~d}$ between pre- and postoperative MRI in CHD patients. However, compared to healthy controls, the patient population showed postoperatively reduced CGM volumes, most likely due to antenatal and postnatal hypoxia and inflammation of the brain in combination with the invasive surgery. This is in agreement with other studies showing smaller volumes already preoperatively (17) of the white matter, gray matter, and cerebellum-and sometimes even antenatally $(18,19)$. CGM shows substantial higher increase over the period of surgery than UWM (21 vs. 5\%), underlining the vulnerability of the cortex in this timeframe. CGM volume reduction seems to be mainly a result of reduced cortical surface area and not of reduced cortical thickness, as the latter 


\section{Articles | Cheesenset tal.}

Table 1. Baseline characteristics

\begin{tabular}{|c|c|c|c|c|}
\hline & & Patients $(n=31)$ & Controls $(n=18)$ & $P$ value \\
\hline Male/female & $n(\%)$ & $23 / 8(74 / 26)$ & $9 / 9(50 / 50)$ & 0.106 \\
\hline Gestational age & Median weeks (range) & $39.29(36.71-41.86)$ & $39.71(37.71-41.14)$ & 0.567 \\
\hline \multirow[t]{2}{*}{ Head circumference at birth } & Median centimeters (range) & $34(32-36)$ & $35.5(33.0-37.5)$ & 0.009 \\
\hline & $n<3$ th percentile $(\%)$ & $2(6.5)$ & $0(0)$ & 0.517 \\
\hline \multirow{4}{*}{ Cardiac abnormality } & With RVOT obstruction & $2(6.5)$ & & \\
\hline & UVH malformation & $8(25.8)$ & & \\
\hline & Left ventricle dominance & $6(19.5)$ & & \\
\hline & Right ventricle dominance & $2(6.5)$ & & \\
\hline Age preoperative MRI & Median gestational age (range) & $40.71(37.86-42.86)$ & & \\
\hline \multirow[t]{3}{*}{ Age postoperative MRI } & Median days (range) & $24(19-52)$ & $23(13-33)$ & 0.087 \\
\hline & Median gestational age (range) & $43.14(40.29-47.14)$ & $42.86(40.86-44.86)$ & 0.178 \\
\hline & Median postoperative days (range) & $13(3-32)$ & N/A & N/A \\
\hline Time between MRI's & Median days (range) & $18(11-42)$ & N/A & N/A \\
\hline \multirow[t]{3}{*}{ Preoperative lesions ${ }^{\mathrm{a}}$} & Total $n(\%)$ & $7(23)$ & N/A & N/A \\
\hline & White matter injury & $6(20)$ & & \\
\hline & Isolated arterial stroke & $3(10)$ & & \\
\hline New postoperative lesions ${ }^{a}$ & $n(\%)$ & $2(7)$ & $\mathrm{N} / \mathrm{A}$ & N/A \\
\hline
\end{tabular}

Baseline characteristics of patients and controls, with the last column showing the comparison between both groups (in $P$ values). Significant $P$ values ( $<0.05)$ have been marked bold. N/A, not applicable; RVOT, right ventricular outflow tract; UVH, univentricular heart malformation.

aThese results can also be found in a previous publication (31).

Table 2. Perioperative characteristics

\begin{tabular}{llc}
\hline & & Patients $(n=31)$ \\
\hline $\begin{array}{l}\text { Mlprostadil } \\
\text { Mean O2 saturation }\end{array}$ & $n(\%)$ & $30(97)$ \\
$\begin{array}{l}\text { Balloon atrioseptostomy } \\
\text { Age at surgery }\end{array}$ & $n(\%)$ & $86(74-96)$ \\
Aortic cross clamp time & Median days (range) & $12(7-62)$ \\
$\begin{array}{l}\text { Extracorporeal circulation } \\
\text { time }\end{array}$ & Median minutes (range) & $201(123-264)$ \\
$\begin{array}{l}\text { Intensive care stay } \\
\text { Postoperative mechanical } \\
\text { ventilation }\end{array}$ & Median days (range) & $7(4-20)$ \\
Hospital stay $(N=30)$ & Median days (range) & $3(1-13)$ \\
\hline
\end{tabular}

Perioperative characteristics of the patient population.

was comparable with healthy controls. The developing brain is particularly vulnerable to disturbances of oxygen and nutrient delivery (9) and due to the cardiac defect, blood supply of the brain can be impaired (20). Fetal studies in patients with CHD suggest hypo-perfusion of the brain already starting in the first or second trimester of pregnancy (18). Additionally, the required $\mathrm{CPB}$ surgery with long anesthesia also leads to suboptimal delivery of oxygen and other substrates during the procedure $(1,4)$. Ischemia and inflammation to the brain influence developing preoligodendrocytes, which are the predominating cells in fetal white matter and known to play an important role in driving cortical development. Quick proliferation and differentiation of these cells under hypoxia creates oligodendrocytes that fail to myelinate the developing axons, which might lead to abnormal cortical maturation (11). This is supported by the finding that the gyrification increase rate appears to be lower in infants suffering white matter injury. This is suggesting an impact of white matter alterations to cortical maturation, which has been explored before in extremely preterm born infants $(11,12)$.

Animal studies have shown significant effects of hypoxia (even when reversible) to cortical gyrification $(21,22)$. Our population, however, did not show a delay in maturation of the cortex, expressed by gyrification index, compared to healthy infants. We did however find smaller cortical volumes and lower gyrification indices in patients with a UVH compared to d-TGA patients. Previous studies on patients with HLHS found lower gyrification indices and immature cortices in fetuses 

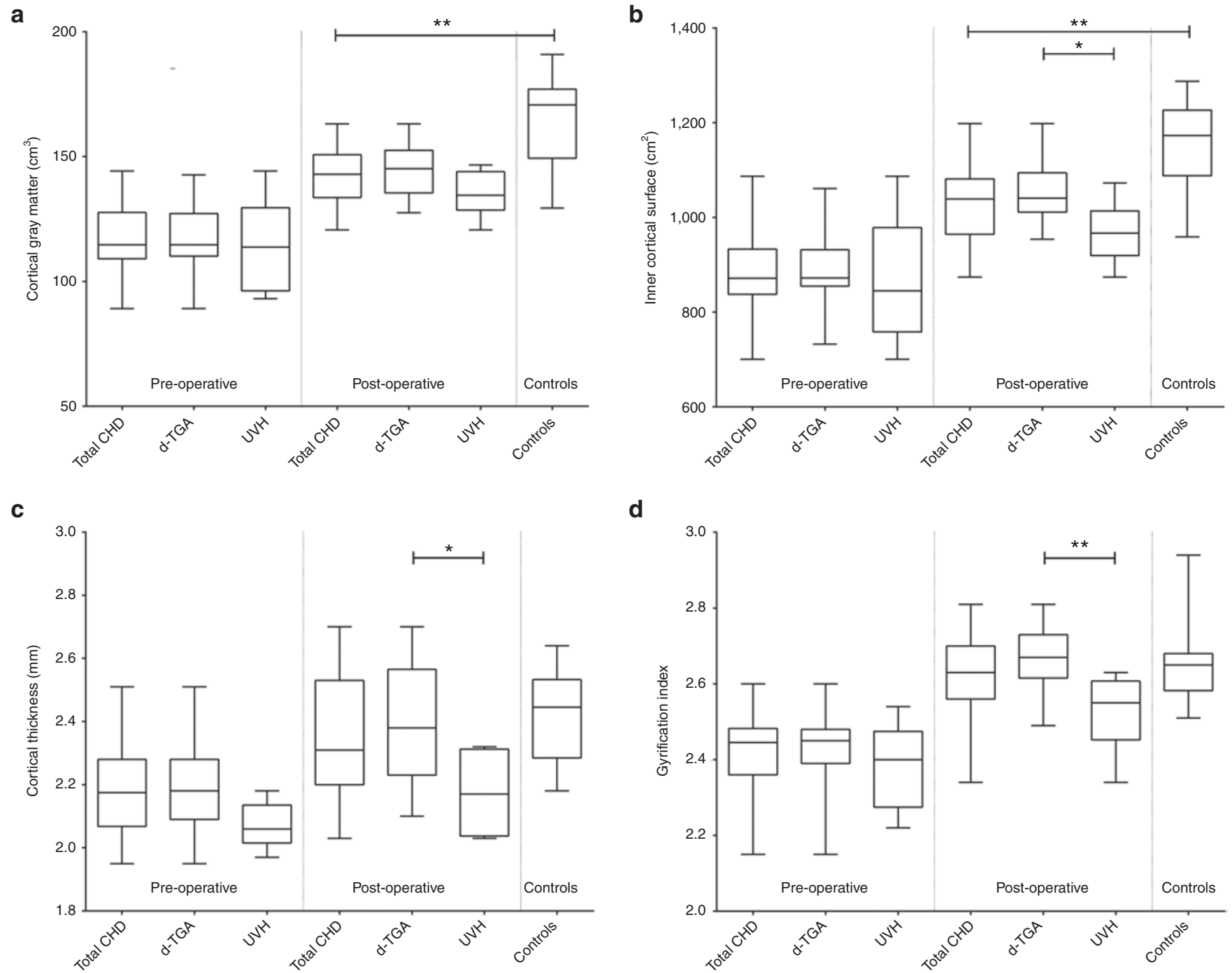

Figure 1. Overview of cortical parameters: The 4 graphs are representing cortical gray matter volume (a), inner cortical surface (b), cortical thickness (c), and gyrification index (d). Per graph boxplots for total congenital heart disease (CHD) population $(n=31)$ preoperative and postoperative are given, as also boxplots for the dextro-transposition of the great arteries subgroup (d-TGA, $n=21$ ), the univentricular heart malformation subgroup (univentricular heart malformation (UVH), $n=8$ ) and controls $(n=18)$, with mean $\pm 1 \mathrm{SD}$ and range. Significant differences between total CHD population and controls, as also significant differences between d-TGA and UVH group are displayed $\left(*<0.05,{ }^{* *}<0.01\right)$.

$(23,24)$ and infants before heart surgery (25). Apparently, alterations of cortical development are most prominent in patients with HLHS and not or less present in patients with d-TGA. We therefore interpret our findings of normal cortical maturation as associated to the less severe CHD diagnoses in our cohort consisting of d-TGA and UVH-opposed to HLHS only in the above-mentioned studies. We did not find significant growth rate differences between the patient subgroups. Previous studies, however, also suggest normal growth rates after surgery in infants with HLHS despite smaller preoperative brain volumes (26). As our control group was only scanned once, no comparison in growth could be made between patients and controls.

We were able to identify several risk factors for altered cortical volume and maturation, which can be objectified in the antenatal period and around birth. Independently from this physiological association, infants with lower weight and/or head circumference at birth had smaller UWM and CGM volumes after birth, which is in agreement with other literature (17). It is of interest that birth weight was a better predictor for altered CGM volume after surgery than head circumference, although intrauterine growth restriction is known to reduce cortex' development in preterms (27). Also the brain sparing effect-lowering cerebral vascular resistance to maximize blood flow to the brain -is described in fetuses with congenital heart disease (20).

Furthermore, patients with a univentricular heart malformation showed reduced cortical volume and delayed gyrification compared to infants with d-TGA. As stated above, biventricular heart defects seem to show less effect to the brain than univentricular heart defects-particularly HLHS (9). Retrograde aortic perfusion via the ductus arteriosus is already present in utero in infants with a hypoplastic left or right heart 
Articles | Claessenset al.

Table 3. Association clinical and cortical parameters

\begin{tabular}{|c|c|c|c|c|c|c|c|c|c|}
\hline \multirow[b]{2}{*}{$\begin{array}{l}\text { Cortical } \\
\text { parameter }\end{array}$} & \multirow[b]{2}{*}{$\begin{array}{c}\text { Time point } \\
\text { (pre or } \\
\text { postoperative) }\end{array}$} & \multicolumn{5}{|c|}{ Perinatal risk factors } & \multicolumn{3}{|c|}{ Preoperative risk factors } \\
\hline & & $\begin{array}{c}\text { Sex (male } \\
\text { vs. female) }\end{array}$ & $\begin{array}{c}\text { Gestational } \\
\text { age }\end{array}$ & $\begin{array}{c}\text { Birth } \\
\text { weight }\end{array}$ & $\begin{array}{c}\text { Head } \\
\text { circumference } \\
\text { at birth }\end{array}$ & $\begin{array}{c}\text { d-TGA vs. } \\
\text { UVH }\end{array}$ & $\begin{array}{l}\text { Balloon } \\
\text { atrioseptostomy } \\
\text { (no vs. yes) }\end{array}$ & $\begin{array}{c}\text { Apgar at } 5 \\
\min \end{array}$ & $\begin{array}{c}\mathrm{O} 2 \\
\text { saturation }\end{array}$ \\
\hline \multirow{2}{*}{$\begin{array}{l}\text { Cortical gray } \\
\text { matter }\left(\mathrm{cm}^{3}\right)\end{array}$} & Pre & - & $0.510^{* *}$ & $0.539^{* *}$ & $0.427^{*}$ & - & - & - & - \\
\hline & Post & $0.390^{*}$ & $0.500^{* *}$ & $0.639 * * a$ & - & - & $0.462^{*}$ & - & - \\
\hline \multirow{2}{*}{$\begin{array}{l}\text { Inner cortical } \\
\text { surface }\left(\mathrm{mm}^{2}\right)\end{array}$} & Pre & - & - & $0.445^{*}$ & $0.539^{* *}$ & - & - & - & - \\
\hline & Post & - & $-{ }^{a}$ & $0.539 * * a$ & $-{ }^{a}$ & $0.495^{*}$ & $0.509 *$ & - & - \\
\hline \multirow[t]{2}{*}{ Thickness (mm) } & Pre & - & - & - & - & - & - & - & - \\
\hline & Post & $0.465^{*}$ & - & - & - & $0.494^{*}$ & - & - & - \\
\hline \multirow[t]{2}{*}{ Gyrification index } & Pre & - & - & - & - & - & - & - & - \\
\hline & Post & $0.534^{* *}$ & $--^{a}$ & $0.416^{*}$ & - & $0.581^{* *}$ & $0.418^{*}$ & - & - \\
\hline \multirow[t]{2}{*}{ White matter $\left(\mathrm{cm}^{3}\right)$} & Pre & $0.427^{*}$ & - & - & $0.547^{* *}$ & - & - & - & - \\
\hline & Post & $0.445^{* a}$ & - & $-{ }^{a}$ & - & - & - & - & - \\
\hline
\end{tabular}

Influence of clinical characteristics (columns) on cortical parameters (rows), with correction for postmenstrual age at MRI. White matter volume was added for comparison. Pearson

correlation coefficients are presented. - = no significant association.

*P value $<0.05$. **P value $<0.01$

a Showed a significant association in the control group. No association between cortical parameters and perioperative risk factors were seen.

UVH, univentricular heart malformation.

resulting in lower cerebrovascular resistance $(20,28)$. A recent study also showed lower cerebral blood flow in infants with a UVH compared to biventricular forms of CHD (29). Finally, the Norwood procedure performed in infants with HLHS is accompanied with more risks for the developing brain than other procedures (1).

Infants requiring preoperative balloon atrioseptostomy had significantly smaller cortical gray matter volumes and lower gyrification indices at postoperative scan than patients who did not require this intervention. Infants in need of BAS show lower preoperative $\mathrm{O}_{2}$ saturation values, which is in line with literature describing higher levels of hypoxemia and hemodynamic instability in those infants. Infants requiring BAS usually are in a worse condition than their peers and seem to be of higher risk for hypoxia and ischemia associated brain injury (30). However, it remains unclear whether our finding of more severe alterations of cortical gray matter development is only an effect of worse hemodynamic condition associated with the need for BAS or whether there is also an effect of the BAS procedure itself. More brain alterations in infants with BAS are also found in studies focusing on focal brain injury and white matter microstructure $(1,4)$, although not confirmed in a previous study with this cohort (31). In our study, performance of BAS was equally prevalent in both d-TGA and UVH patients, which reduced the risk of confounding. The high incidence of BAS in this small UVH population might be by chance. However, other factors indicating severity of illness (preoperative oxygen saturation, Apgar score, days of mechanical ventilation, and total duration of hospital stay) were not found to be related to cortical development. Preoperative saturation has been reported as a risk factor for qualitative white matter brain injury $(1,31)$, but could not be found as a risk factor for growth and maturation of the cortex in this study.
The first limitation of this study is the small (heterogenous) population leading to limited statistical strength. Second, only MR images of healthy controls at comparable postoperative PMA were obtained (as it was not considered ethical to perform two MRI scans in healthy neonates). Therefore, no comparison of cortical volumes and maturation before surgery or growth could be made, which would be of interest when examining the effect of surgery to the delay in cortex development. Third, the cortical measurements were acquired fully automatically following manual segmentation. Limitations of this method, although of minor importance, can be found in a previously published article (32). This method requires sufficient quality of the MR images, which induced loss of data in this population (preoperatively five cases and postoperatively eight cases). However, selection bias does not seem to play a role, as baseline characteristics were found to be similar between both groups. Unfortunately, this method did not allow regional cortical measurements in this cohort which would be of interest in future populations. Finally, the low incidence of brain injury in this patient group should be noted, which could be influenced by the scanning protocol ( $1.5 \mathrm{~T}, 2.5 \mathrm{~mm}$ slice thickness) and other contributors (31).

Infants with congenital heart disease show a significant increase of cortical volume and gyrification from the pre- to postoperative period, but cortical volume remains reduced after surgery when compared to healthy term born controls. Gyrification is delayed in neonates with $\mathrm{UVH}$, but not in d-TGA. Lower birth weight and preoperative requirement of balloon atrioseptostomy are independent risk factors for impaired postoperative cortical volume and gyrification. Lower gestational age is a risk factor for smaller cortical volume but not for delay in maturation. The cortex plays an important role in cognitive, motor and behavioral functioning and delay in cortical development in infants with severe 
CHD might lead to alterations in these neurodevelopmental domains later in life. The clinical risk factors should be confirmed in a larger cohort with preoperative controls, and prove their functional relevance by investigating their association to (long-term) neurodevelopmental outcome.

\section{METHODS}

\section{Study Design}

This cohort is part of an ongoing prospective cohort study (Heart and Brain Research Group Zurich) on cerebral injury and neurodevelopmental outcome in infants with CHD treated at the University Children's Hospital in Zurich. Results on brain injury (31) and postoperative brain volumes (15) have been published previously. The study has been approved by the Ethical Committee of the Canton Zurich and written parental informed consent was obtained.

\section{Patients}

Infants born between November 2009 and January 2012 with severe CHD requiring surgery under full-flow $\mathrm{CPB}$ were enrolled. Cardiac diagnoses included dextro-transposition of the great arteries (d-TGA), univentricular heart malformation (UVH, including hypoplastic left heart syndrome (HLHS)), and coarctation of the aorta (CoA). Excluded were those born with a gestation of less than $36 \mathrm{wk}$ or with a genetic syndrome. Detailed information on the surgical procedure and management can be found in a previous article (31). In addition, between January 2011 and January 2012 healthy term born infants were prospectively recruited from the postnatal ward of the University Hospital Zürich for MRI of the brain. These control infants were not admitted to the neonatal unit, did not show cerebral injury on MRI and had normal neurodevelopmental outcomes at $1 \mathrm{y}$ of age.

\section{MR Imaging}

All infants underwent an MRI of the brain before and after surgery. The healthy control infants were scanned once, at corresponding postoperative postmenstrual age. MRI was performed on a 3.0 T MR scanner (SignaHDxt, GE Healthcare, Milwaukee, WI) using T2-weighted fast-spin-echo sequences in three planes (slice thickness $=2.5 \mathrm{~mm}$, $\mathrm{TR}=5,300 \mathrm{~ms}, \mathrm{TE}=102 \mathrm{~ms}$ ). More information on scanning protocol and definition of brain injury can be found in a previous publication (31).

\section{Cortical Morphologic Measurements}

For all infants the axial T2-weighted images were manually segmented into UWM and CGM (15). Based on these segmentations, UWM and CGM volume (in $\left.\mathrm{cm}^{3}\right)$, inner cortical surface $\left(i \mathrm{CS}\right.$, in $\left.\mathrm{cm}^{2}\right)$, cortical thickness (in $\mathrm{mm}$ ), and GI were computed using the approach described in a recently published paper (32). The iCS is the surface of the border between UWM and CGM. The gyrification index was measured as the ratio between iCS and a smooth hull around the white matter (i.e., a simulation of a smooth cortex without any folds). The volumetric, surface and thickness measurements were used as quantitative considerations of cortical volume and cortical growth. Gyrification index was used to interpret the level of cortical maturation (or cortical folding). All parameters together will be referred to as cortical parameters with cortical development considered as the combination of cortical growth and maturation.

\section{Statistical Analysis}

Statistical analysis was performed using IBM SPSS statistics ${ }^{\circledR}$ version 21.0. An independent $t$-test was used to describe the differences in clinical characteristics and cortical parameters between patients and controls (with a $\chi^{2}$ test for sex), a paired $t$-test for comparison of preand postoperative cortical parameters. All cortical parameters were corrected for PMA at scan, as this effect was not subject of investigation. Absolute growth (corrected for days between scans) was calculated as a difference between pre- and postoperative scan, relative growth as a percentage of increase. Linear regression analysis was performed to examine separately the associations between the clinical risk factors (as independent variables) and cortical parameters (as dependent variables). This analysis was repeated with correction for sex. As the sample size of this study was small and also the aim was to explore clinical risk factors, a multivariable regression analysis was not performed. A $P$ value $<0.05$ was considered significant.

\section{ACKNOWLEDGMENTS}

We thank all members of the Research Group Heart and Brain at the University Children's Hospital Zurich. Special thank goes to the study nurses: Rabia Liamlahi, Klaudija Batinic, and Sonia Bertholdt. Further Members of the Heart and Brain research group are: Felix H. Sennhauser, Rabia Liamlahi, Oliver Kretschmar, Hitendu Dave, Barbara R. Plecko, Christian Kellenberger, Vera Bernet, Christoph Bürki and René Prêtre. We thank the Division of Obstetrics and the Department of Neonatology (in particular Cornelia Hagmann) of the University Hospital Zurich for the support and cooperation in recruiting the control group.

\section{STATEMENT OF FINANCIAL SUPPORT}

This study is supported by the Swiss Heart Foundation, Else Kröner-Fresenius-Stiftung. The sponsors had no influence on study design, collection, analysis, and interpretation of data, the writing of the report, or the decision to submit the paper for publication. The first draft of the manuscript has been written by N. Claessens. The authors have no financial relationships or conflict of interest to disclose.

\section{REFERENCES}

1. McQuillen PS, Barkovich AJ, Hamrick SE, et al. Temporal and anatomic risk profile of brain injury with neonatal repair of congenital heart defects. Stroke 2007;38(2 Suppl):736-41.

2. Snookes SH, Gunn JK, Eldridge BJ, et al. A systematic review of motor and cognitive outcomes after early surgery for congenital heart disease. Pediatrics 2010;125:e818-27.

3. Majnemer A, Limperopoulos C, Shevell M, Rohlicek C, Rosenblatt B, Tchervenkov C. Developmental and functional outcomes at school entry in children with congenital heart defects. J Pediatr 2008;153:55-60.

4. Dimitropoulos A, McQuillen PS, Sethi V, et al. Brain injury and development in newborns with critical congenital heart disease. Neurology 2013;81:241-8.

5. Ortinau C, Beca J, Lambeth J, et al. Regional alterations in cerebral growth exist preoperatively in infants with congenital heart disease. J Thorac Cardiovasc Surg 2012;143:1264-70.

6. Mlczoch E, Brugger P, Ulm B, et al. Structural congenital brain disease in congenital heart disease: results from a fetal MRI program. Eur J Paediatr Neurol 2013;17:153-60.

7. Berman JI, Hamrick SE, McQuillen PS, et al. Diffusion-weighted imaging in fetuses with severe congenital heart defects. AJNR Am J Neuroradiol 2011;32:E21-2.

8. Algra SO, Jansen NJ, van der Tweel I, et al. Neurological injury after neonatal cardiac surgery: a randomized, controlled trial of 2 perfusion techniques. Circulation 2014;129:224-33.

9. McQuillen PS, Miller SP. Congenital heart disease and brain development. Ann N Y Acad Sci 2010;1184:68-86.

10. Miller SP, McQuillen PS, Hamrick S, et al. Abnormal brain development in newborns with congenital heart disease. N Engl J Med 2007;357: 1928-38.

11. Volpe JJ. Brain injury in premature infants: a complex amalgam of destructive and developmental disturbances. Lancet Neurol 2009;8:110-24.

12. Back SA, Miller SP. Brain injury in premature neonates: A primary cerebral dysmaturation disorder? Ann Neurol 2014;75:469-86.

13. Clouchoux C, Kudelski D, Gholipour A, et al. Quantitative in vivo MRI measurement of cortical development in the fetus. Brain Struct Funct 2012;217:127-39.

14. Dubois J, Benders M, Borradori-Tolsa C, et al. Primary cortical folding in the human newborn: an early marker of later functional development. Brain 2008;131(Pt 8):2028-41.

15. Von Rhein M, Buchmann A, Hagmann C, et al. Severe congenital heart defects are associated with global reduction of neonatal brain volumes. J Pediatr 2015;1-6.

16. Dubois J, Benders M, Cachia A, et al. Mapping the early cortical folding process in the preterm newborn brain. Cereb Cortex 2008;18: 1444-54. 


\section{Articles | Claessenset tal.}

17. Owen M, Shevell M, Donofrio M, et al. Brain volume and neurobehavior in newborns with complex congenital heart defects. J Pediatr 2014;164: 1121-1127.e1.

18. Schellen C, Ernst S, Gruber GM, et al. Fetal MRI detects early alterations of brain development in Tetralogy of Fallot. Am J Obstet Gynecol 2015;213:392.e1-7.

19. Zeng S, Zhou Q, Zhou J, Li M, Long C, Peng Q. Impaired brain growth of intracranial structures in fetuses with complex congenital heart disease as measured by 3D ultrasonography. Ultrasound Obstet Gynecol 2015;46:174-81.

20. Donofrio MT, Bremer YA, Schieken RM, et al. Autoregulation of cerebral blood flow in fetuses with congenital heart disease: the brain sparing effect. Pediatr Cardiol 2003;24:436-43.

21. Rees S, Stringer M, Just Y, Hooper SB, Harding R. The vulnerability of the fetal sheep brain to hypoxemia at mid-gestation. Brain Res Dev Brain Res 1997;103:103-18.

22. Dean JM, McClendon E, Hansen K, et al. Prenatal cerebral ischemia disrupts MRI-defined cortical microstructure through disturbances in neuronal arborization. Sci Transl Med 2013;5:168ra7.

23. Clouchoux C, du Plessis AJ, Bouyssi-Kobar M, et al. Delayed cortical development in fetuses with complex congenital heart disease. Cereb Cortex 2013;23:2932-43.

24. Glauser TA, Rorke LB, Weinberg PM, Clancy RR. Congenital brain anomalies associated with the hypoplastic left heart syndrome. Pediatrics 1990;85:984-90.
25. Ortinau C, Alexopoulos D, Dierker D, Van Essen D, Beca J, Inder T. Cortical folding is altered before surgery in infants with congenital heart disease. J Pediatr 2013;163:1507-10.

26. Ortinau C, Inder T, Lambeth J, Wallendorf M, Finucane K, Beca J. Congenital heart disease affects cerebral size but not brain growth. Pediatr Cardiol 2012;33:1138-46.

27. Tolsa CB, Zimine S, Warfield SK, et al. Early alteration of structural and functional brain development in premature infants born with intrauterine growth restriction. Pediatr Res 2004;56:132-8.

28. Kaltman JR, Di H, Tian Z, Rychik J. Impact of congenital heart disease on cerebrovascular blood flow dynamics in the fetus. Ultrasound Obstet Gynecol 2005;25:32-6.

29. Nagaraj UD, Evangelou IE, Donofrio MT, et al. Impaired global and regional cerebral perfusion in newborns with complex congenital heart disease. J Pediatr 2015;167:1018-24.

30. McQuillen PS, Hamrick SE, Perez MJ, et al. Balloon atrial septostomy is associated with preoperative stroke in neonates with transposition of the great arteries. Circulation 2006;113:280-5.

31. Bertholdt S, Latal B, Liamlahi R, et al.; Research Group Heart and Brain. Cerebral lesions on magnetic resonance imaging correlate with preoperative neurological status in neonates undergoing cardiopulmonary bypass surgery. Eur J Cardiothorac Surg 2014;45:625-32.

32. Moeskops P, Benders MJ, Kersbergen KJ, et al. Development of cortical morphology evaluated with longitudinal MR brain images of preterm infants. PLoS One 2015;10:e0131552. 